\title{
Author Correction: Hidden hassium
}

Michael A. Tarselli

Correction to: Nature Chemistry https://doi.org/10.1038/s41557-018-0037-4, published online 22 March 2018.

In this In Your Element originally published, there were several inaccuracies and the main text has been revised accordingly: in paragraph 1, the sentence starting "Among the already little-known..., has been revised to: "Among the already little-known super-heavies, element 108 doesn't have the controlled reactivity of a seaborgium atom, or the relative stability of fermium - the longest-lived isotope is ${ }^{257} \mathrm{Fm}$ with a net 100 day half-life, and ${ }^{252} \mathrm{Fm}$ is believed to resist spontaneous fission rather well ${ }^{1}$, though it undergoes $\alpha$-decay." The final two sentences of paragraph 2 have been revised to "This approach, however, only works up to element 100 (fermium). The team at the Joint Institute for Nuclear Research (JINR) in Dubna, USSR (now Russia), led by actinide heavyweight Yuri Oganessian, then pioneered the techniques of 'cold' fusion (collisions of two early elements such as iron and bismuth) and 'hot' fusion (using actinide radionuclides as the targets)." In the first sentence of paragraph 3, "First, a heavy target such as einsteinium or plutonium is bombarded..." has been revised to "In hot fusion, researchers bombard a heavy target such as einstinium or plutonium..."; and in the third sentence, "new accelerators" was changed to "new equipment" and "pushing back the limit to 114" was changed to "pushing back the limit to 118...so far". In paragraph 4, the sentence beginning "Meitnerium was adopted..." has been revised to "Meitnerium was adopted, but for 108 the suggestion of the German team, led by Peter Armbruster and Gottfried Münzenberg, was the one ratified in 1997: hassium, which honours the German state of Hesse (whose map and coat of arms are pictured).”. Finally, in paragraph 5, the line "specialized detectors had to be built." was clarified to read: "specialized detectors were built to study its characteristic $\alpha$-decay and probe its reaction chemistry."; and at the end of the paragraph, "a measurable amount of" has been removed from the final sentence, and "presumably $\mathrm{HsO}_{4}$." has been changed to: "presumably $\mathrm{HsO}_{4}$, whose single ${ }^{269} \mathrm{Hs}$ atom was identified by $\alpha$-decay"."

Published online: 4 July 2018

https://doi.org/10.1038/s41557-018-0108-6

\section{Author Correction: Dynamic actuation of glassy polymersomes through isomerization of a single azobenzene unit at the block copolymer interface}

\author{
Mijanur Rahaman Molla, Poornima Rangadurai, Lucas Antony (D), Subramani Swaminathan, Juan J. de Pablo \\ and S. Thayumanavan (D)
}

Correction to: Nature Chemistry https://doi.org/10.1038/s41557-018-0027-6, published online 30 April 2018.

In the version of this Article originally published, multiple changes to the "Results and discussion" section were required. In paragraph 1, "(Supplementary Fig. 1)" should have read "(Fig. 1e-j and Supplementary Fig. 1)"; in the first sentence of paragraph 3, "(R6G)" should have read "(R6G, Fig. 2i)"; in paragraph 6 in the sentence beginning "Temporal release of hydrophilic..., Supplementary Fig. 4 should have been cited after " $360 \mathrm{~nm}$ "; in paragraph 9, in the sentence beginning "To test this..., "Fig. 4e" should have read "Fig. 4a"; in paragraph 10, in the sentence beginning "When the irradiation..., "(Fig. 4a-d)" should have read "(Fig. 4d,e)"; in paragraph 11, in the sentence beginning "Pristine PLA", "P1" should have read "P2"; and in the penultimate paragraph, in the sentence beginning "Moreover, a control PEG-PLA...", "block copolymer" should have been followed by (P5); Fig. 4g should have been Fig. 4c; "hydrophobic azobenzene small molecules" should have been followed by (12); and Fig. 4f should have been Fig. 4b. Finally, Supplementary Videos 1 and 2 were missing from the Article. All of these corrections have been made to the online versions.

Published online: 28 June 2018

https://doi.org/10.1038/s41557-018-0090-z 